\title{
Construction Classification -Approach in Small Societies
}

\author{
Roode Liias* \\ Tallinn University of Technology, Europe
}

*Corresponding author: Roode Liias, Tallinn University of Technology, Estonia, Europe.

\section{Opinion}

Much is written and discussed about the problems of and in the construction sector, especially due to very low efficiency level compared to all the other industries/businesses. Accordingly, different improvement possibilities are proposed - starting from engineering improvements of the construction technology and ending up with reasonable restructuring of the management schemes and methods used in the sector. No universal and miracle cure has been neither proposed by the academicians nor invented and introduced in business in practice, especially on the sites. Digitalization is currently considered perhaps as one of the key preconditions to start with to increase the efficiency. The magical acronym BIM is currently used in most of the academic papers and even politicians have started to advocate it - this the only right way we have to go! Information is in the central position in the word BIM joining the buildings (B) and management (M) or modelling. Certainly, information is the cornerstone when solving several problems of the sector. But very often it is repeated, that information has value only when it is available at right time and in a right place. Shortly - clearly and easily accessible for all the users. In a small country like Estonia with less than 1.5 mil inhabitants it may seem there are no borders for communication and information exchange. But for years we have targeted now to improve especially the information model of the whole construction and property sector. The quite fragmented usage of digital tools on only some stages (e.g. by the designers or by manufacturers) of the lifecycle do not help much to improve the general situation when the others (e.g. clients and maintenance staff) are not involved in this information flow. The starting point here is the so-called concept of introducing the "common language" - introducing the unified classification system for the construction and property/facilities sector. There are numerous construction classification systems already proposed world-wide, several of which are already old- fashioned and fragmented and not suitable for integrated digital usage anymore. The others are rather contemporary ones, but too much regulated and fixed solutions, not allowing the national legal and cultural peculiarities to be considered.

The topic of classification in the construction industry is not new by any means. In 2018 Estonian Ministry of Economic Affairs and Communication called for a tender to develop a uniform classification system for construction, which will take into account the current trends in BIM technology and digital construction and creates a uniform and comprehensible language for the management of construction information. This uniform classification system shall cover the entire life cycle of the building, from the initial task to the environmentally sound demolition. The uniform classification system shall be applicable to both buildings and infrastructure installations. The common classification system must allow the creation of a comprehensible digital information space for all participants in the life cycle of a building. The system must provide a clear, transparent and rational structure, which can result in the implementation of BIM technology and workflow based on it, simplify the exchange of information, information processing and organization of activities, i.e. construction communication. In parallel to national studies Estonia and several small neighboring societies have started to cooperate in finding and establishing a unified construction classification system covering the whole lifecycle in the construction sector and suitable for these national construction markets. Reasonable success has been gained especially when initiating the cross-border cooperation between the societies with different national languages and legislations, but still great many constraints appear. Though English has become the common language for international cooperation and for preparing the management documents in the sector, but now it appears that there are different interpretations of these terms into different national languages. Rather often (e.g. when using FIDIC documents) 
English is considered the governing language that is the bases for all the translations, interpretations and when solving the disputes, but now English is only the language for communication for the professionals. Each national language has its own traditional terminology, which is quite often fixed and described also in the relevant legal acts, and therefore this has to be considered as the priority when structuring the classification system. It seems that developing a suitable classification system has become a process of standardizing the principles included already in different standards either in the international or in the national ones. The ISO $120006-$ 2:2015 (Building construction -- Organization of information about construction works -- Part 2: Framework for classification) gives currently the most acceptable and widely followed framework for construction classification. One thing is the conception, but more serious issues arise when one has to furnish the "boxes" (the classes) of the Figure 1- "Classes and the general relationship between them" of this Standard there. Different workgroups for ISO and CEN have compiled standards on quite similar topics but following different methodological approaches. How to find a common agreement and understanding in these situations between different national workgroups? There is no single and quick approach possible only time and relevant discussions between the stakeholders are required here (Figure 1).

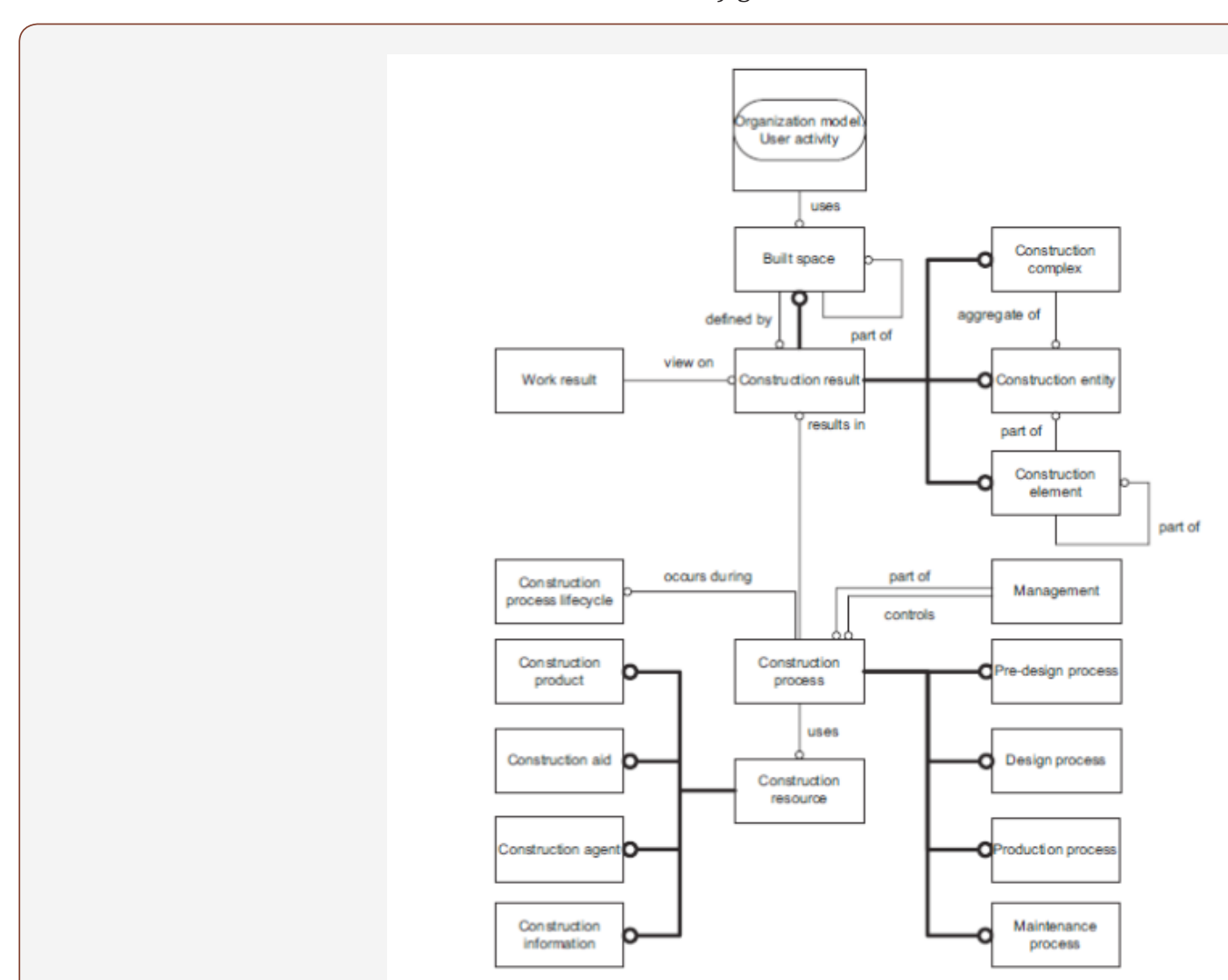

Figure 1: Classes and the general relationship between them.

But the real problem in this cooperation is the human factor. When classifying anything most of the people are used to classical hierarchic systems that have been used for centuries in most of the fields of activities people are involved to, incl. construction. But the logic and principles of usage of faceted classification systems creates problems both for understanding and for implementing. Here we as the academic sector representatives find that certain number of academic hours is required to address for the principles of classification in the academic classes as well. To address the sector and the professionals one has to start from the students. So, the development of a national classification system requires also serious academic input Finalizing. We fully accept the professional quality of the existing and world-wide known construction classification systems and their role as the serious management tools for the sector. But still small national markets need their specific systems to use and develop the national terminology, culture and follow the national legislation principles there. Everybody understands that there are no free of charge "sandwiches". But how to upgrade the system and to provide the relevant software and guideline documents for free (or at least for affordable price) for all the users. Really, it is quite challenging to reach the goals stated there is the need for a unified system that will guarantee the quality of information exchange.

\section{Acknowledgment}

None.

\section{Conflict of Interest}

No conflict of interest. 